\title{
ANÁlISE POR PLANEJAMENTO EXPERIMENTAL DOS FATORES QUE INFLUENCIAM A EXTRAÇÃO DO CORANTE DA CASTANHOLA
}

\author{
V. H. UCHIDA ${ }^{1}$, T. B. V. MIGUEL ${ }^{2}$, A. L. M. MATA ${ }^{3}$, M. M. L. DUARTE ${ }^{3}$ \\ ${ }^{1}$ Universidade Federal do Rio Grande do Norte, Programa de Pós-Graduação em Engenharia \\ Química \\ ${ }^{2}$ Universidade Federal do Rio Grande do Norte, Departamento de Engenharia de Alimentos \\ ${ }^{3}$ Universidade Federal do Rio Grande do Norte, Departamento de Engenharia Química \\ E-mail para contato: vivi_hiromi@yahoo.com.br
}

RESUMO - A Terminalia catappa Linn, árvore conhecida como castanhola, possui frutos que são comestíveis e fonte de proteínas e lipídeos. Estudos anteriores comprovam também a presença de antocianinas, que são compostos pertencentes ao grupo dos flavonóides. Estes possuem um papel fundamental na habilidade para capturar radicais livres e assim, promover a prevenção de várias doenças. $\mathrm{O}$ objetivo do presente trabalho foi avaliar os fatores que influenciam a extração do corante da castanhola (temperatura, $\mathrm{pH}$, tempo e relação do solvente), adotando o planejamento fatorial $2^{4}$. As variáveis resposta foram a atividade antioxidante (AA), o teor de compostos fenólicos totais (CFT) e o teor de antocianinas monoméricas totais (AMT) e o software utilizado foi o Statistica 7.0. O fruto foi triturado e a polpa obtida foi submetida a uma extração em um reator encamisado, tipo batelada, acoplado a um banho termostático com velocidade de agitação e temperatura controláveis, usando como solvente uma relação entre água e etanol. As amostras foram analisadas por espectrofotometria. Dos fatores estudados o $\mathrm{pH}$ foi o que mostrou mais influência na extração em relação às variáveis resposta, apresentando o melhor rendimento da extração em um valor de 3,5 .

\section{INTRODUÇÃO}

A Terminalia catappa Linn, da família Combretaceae, cresce em regiões tropicais e subtropicais, particularmente localizadas em áreas costeiras. Essa espécie é nativa de áreas próximas a regiões costeiras do Oceano Índico, na Ásia tropical e da região que compreende várias ilhas a oeste do Oceano Pacífico, como Malásia, Indonésia e ilhas da região da Melanésia (TEIXEIRA, 2010). Os frutos da castanhola possuem uma polpa carnosa, contendo em seu interior uma semente arredondada e rica em óleo, envolvida por uma casca muito dura. A 
coloração do fruto é verde passando ao amarelo e vermelho quando maduros. Eles são comestíveis, fonte de proteínas e lipídios e são utilizados em alimentos, especialmente para as crianças, aves e outros animais.

A pigmentação natural presente no fruto da castanhola indica a presença de antocianinas, componentes de natureza fenólica, pertencentes ao grupo dos flavonóides, que apresentam atividade antioxidante. Os flavonóides possuem um papel fundamental na habilidade para capturar radicais livres e, assim, promover a prevenção de várias doenças.

O objetivo do presente trabalho foi avaliar os fatores que influenciam a extração do corante da castanhola (temperatura, $\mathrm{pH}$, tempo e relação do solvente), adotando o planejamento fatorial de $2^{4}$. As variáveis resposta foram a atividade antioxidante (AA), o teor de compostos fenólicos totais (CFT) e o teor de antocianinas monoméricas totais (AMT). O software utilizado foi o Statistica 7.0.

\section{MATERIAL E MÉTODO}

Para a extração do corante utilizou-se o pó da polpa da fruta. Para isso, a polpa foi cortada em pedaços grandes e submetida a uma secagem em uma estufa com ventilação forçada a temperatura de $70^{\circ} \mathrm{C}$ por 1 hora. Em seguida a polpa foi triturada, pesada, e levada novamente à estufa, pesando-se a cada 15 min até o peso permanecer constante. A polpa foi triturada novamente e peneirada para obtenção do pó a ser utilizado na extração do corante (Figura 1).

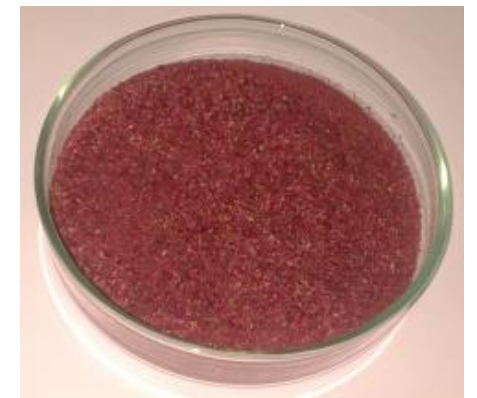

Figura 1 - Pó da polpa de castanhola

Com o planejamento fatorial $2^{4}$, com 16 experimentos e 4 pontos centrais (PC), foi possível a verificação da influência dos parâmetros na obtenção de extrato, em relação ao teor dos compostos extraídos. Para este sistema, foi levada em consideração 4 variáveis independentes: temperatura (T), relação de solventes, água/etanol (v/v), tempo (t) e pH. Os fatores avaliados nos planejamentos e seus respectivos níveis, em valores usados na extração, são apresentados na Tabela 1. 
Tabela 1 - Fatores avaliados nos planejamentos e seus respectivos níveis.

\begin{tabular}{|c|c|c|c|}
\hline Fatores & - & $\mathbf{0}$ & + \\
\hline $\mathbf{T}\left({ }^{\mathbf{0}} \mathbf{C}\right)$ & 25 & 37,5 & 50 \\
\hline $\mathbf{t}$ (min) & 10 & 20 & 30 \\
\hline v/v (concentração de água) & 25 & 37,5 & 50 \\
\hline $\mathbf{p H}$ & 3,5 & 5,5 & 7,5 \\
\hline
\end{tabular}

As extrações foram realizadas em um reator enjaquetado, acoplado a um banho termostato, com temperatura e agitação controláveis, conforme apresentado na Figura 2.

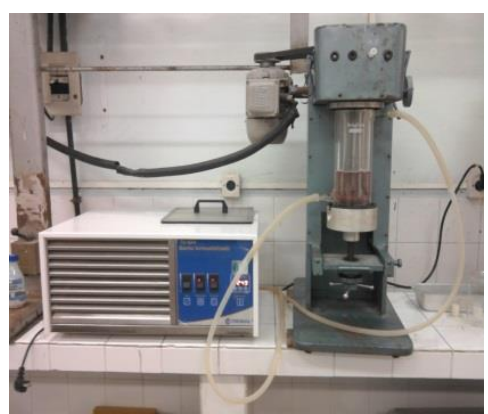

Figura 2- Reator enjaquetado acoplado a um banho termostatizado

As concentrações de CFT nos extratos foram obtidas através do método do FolinCiocalteau, conforme Cheplick et al. (2010). As AMT foram quantificadas segundo o método de Giusti e Wrolstad (2001), pelo método do $\mathrm{pH}$ diferencial. A AA foi determinada pelo método de Duarte-Almeida et al. (2006), através do sequestro do radical livre DPPH•.

\section{RESULTADOS E DISCUSSÕES}

De acordo com o planejamento experimental proposto foram realizados aleatoriamente um total de 16 experimentos com 4 pontos centrais. A Tabela 2 apresenta todas as combinações feitas pelo planejamento fatorial e seus respectivos valores na obtenção da atividade antioxidante (AA), compostos fenólicos totais (CFT) e antocianinas monoméricas totais (AMT).

Tabela 2- Matriz experimental da extração e resultados obtidos.

\begin{tabular}{|l|c|c|c|c|c|c|c|}
\hline Ensaios & T $\left({ }^{0} \mathbf{C}\right)$ & v/v & Tempo (min) & pH & AA & CFT & AMT \\
\hline
\end{tabular}


19 a 22 de outubro de 2014

Florianópolis/SC

\begin{tabular}{|c|c|c|c|c|c|c|c|}
\hline $\mathbf{1}$ & 1 & 1 & 1 & 1 & 15,58238 & 6365,267 & 88,61542 \\
\hline $\mathbf{2}$ & -1 & 1 & 1 & 1 & 28,60107 & 3415,33 & 80,3773 \\
\hline $\mathbf{3}$ & 1 & -1 & 1 & 1 & 28,31656 & 2767,623 & 92,90146 \\
\hline $\mathbf{4}$ & -1 & -1 & 1 & 1 & 28,96471 & 3548,673 & 76,64788 \\
\hline $\mathbf{5}$ & 1 & 1 & -1 & 1 & 25,07012 & 2268,842 & 53,9374 \\
\hline $\mathbf{6}$ & -1 & 1 & -1 & 1 & $\mathbf{2 9 , 7 4 4 3 8}$ & 5142,251 & 75,47896 \\
\hline $\mathbf{7}$ & 1 & -1 & -1 & 1 & 29,21045 & 5089,358 & $-86,3332$ \\
\hline $\mathbf{8}$ & -1 & -1 & -1 & 1 & 27,55283 & 13564,58 & 78,45693 \\
\hline $\mathbf{9}$ & 1 & 1 & 1 & -1 & 17,47112 & $\mathbf{3 0 3 0 7 , 5 3}$ & 203,0584 \\
\hline $\mathbf{1 0}$ & -1 & 1 & 1 & -1 & 27,34704 & 14958,14 & 163,4264 \\
\hline $\mathbf{1 1}$ & 1 & -1 & 1 & -1 & 14,09663 & 26106,80 & $\mathbf{3 7 6 , 3 9 2 9}$ \\
\hline $\mathbf{1 2}$ & -1 & -1 & 1 & -1 & 23,37434 & 20668,5 & 218,3101 \\
\hline $\mathbf{1 3}$ & 1 & 1 & -1 & -1 & 22,20719 & 23274,35 & 209,6267 \\
\hline $\mathbf{1 4}$ & -1 & 1 & -1 & -1 & 18,32852 & 20106,23 & 213,1891 \\
\hline $\mathbf{1 5}$ & 1 & -1 & -1 & -1 & 6,571019 & 23361,5 & 177,5648 \\
\hline $\mathbf{1 6}$ & -1 & -1 & -1 & -1 & 21,8257 & 18324,1 & 151,4032 \\
\hline $\mathbf{P C}$ & 0 & 0 & 0 & 0 & 23,98019 & 14034,21 & 161,4226 \\
\hline $\mathbf{P C}$ & 0 & 0 & 0 & 0 & 17,96047 & 17302,42 & 119,0074 \\
\hline $\mathbf{P C}$ & 0 & 0 & 0 & 0 & 23,98019 & 14034,21 & 161,4226 \\
\hline $\mathbf{P C}$ & 0 & 0 & 0 & 0 & 22,96644 & 18302,42 & 169,9077 \\
\hline
\end{tabular}

Os resultados obtidos foram avaliados pelo software Statistic 7.0, utilizando o erro puro através de 4 pontos centrais. Desta forma, o erro experimental foi considerado independente do erro do modelo matemático. Os valores dos coeficientes de determinação $\left(\mathrm{R}^{2}\right)$ de cada modelo matemático gerado foram de 94,68\%, 97,03 e 98,26 para a atividade antioxidante, compostos fenólicos totais e antocianinas monoméricas totais respectivamente.

Dentre as condições avaliadas, o maior rendimento do extrato para AA, CFT e AMT, foi alcançado nos ensaios 6, 9 e 11, respectivamente, com 29,74 $\mu \mathrm{mol}$ Trolox eq/g da amostra para a atividade antioxidante, 30307,53 $\mathrm{mg}$ GAE/100g da amostra para o teor de compostos fenólicos totais e 376,39 mg ci-3-gli/100 g para a concentração de antocianinas monoméricas totais. Os gráficos de Pareto para as variáveis resposta são apresentados nas Figuras 3, 5 e 6 .

Na análise do diagrama de Pareto (Figura 3) para compostos fenólicos totais, observou-se o pH bastante significativo e, em menor significância, a interação da Temperatura (1) e pH (4).

Azevedo (2010) também analisou que as condições ótimas de operação para a obtenção de compostos fenólicos estão na faixa de temperatura entre $50^{\circ} \mathrm{C}$ a $60^{\circ} \mathrm{C}$. 


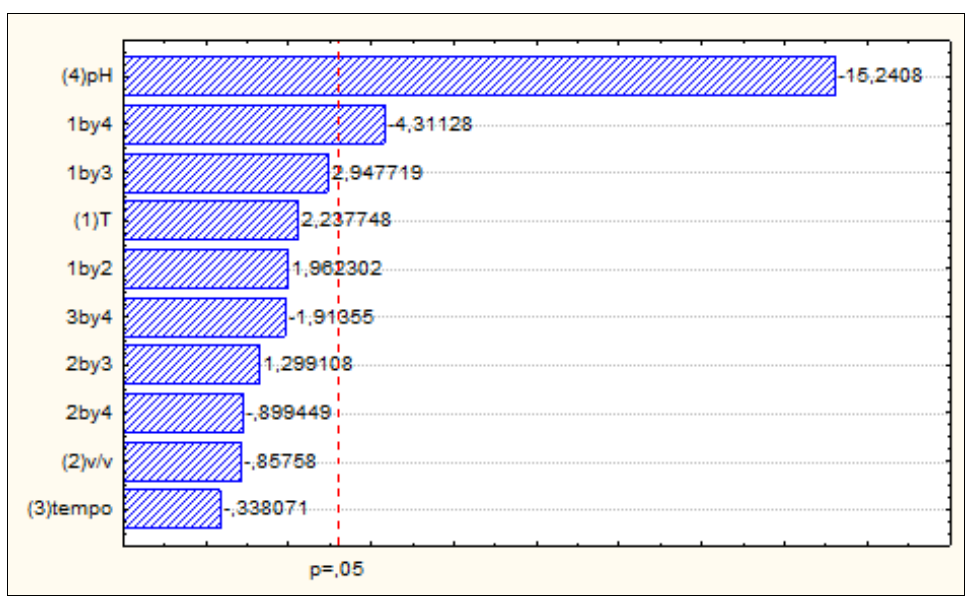

Figura 3 - Diagrama de Pareto para compostos fenólicos totais

Na Figura 4, observando a curva de nível, constata-se que as condições ótimas para obtenção dos CFT estão com valores de temperatura maiores e um menor pH. Segundo Jing e Giusti (2007), temperaturas mais altas aumentam a difusão e solubilidade do composto e diminuem a viscosidade de solventes, resultando assim na melhoria da extração.

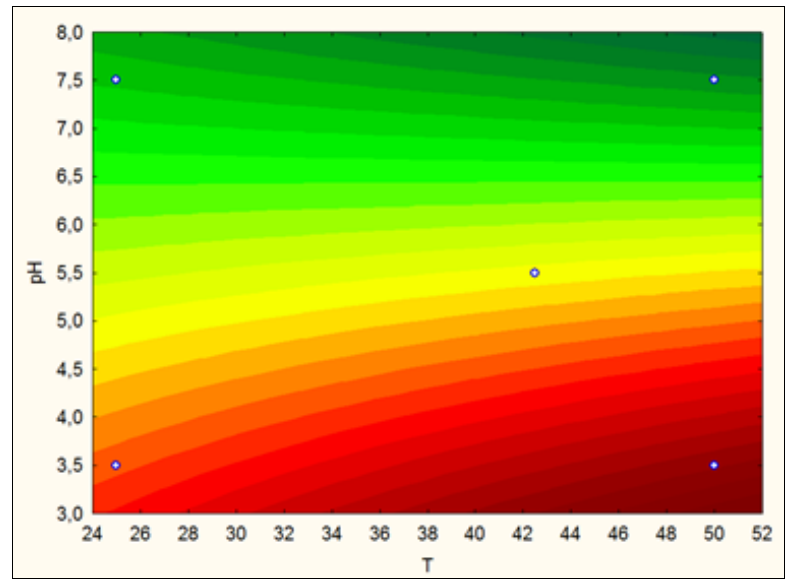

Figura 4 - Curva de nível para CFT

Para a atividade antioxidante foi observado que o $\mathrm{pH}$, temperatura e a interação da temperatura, relação de solventes e $\mathrm{pH}$ foi pouco significativo (Figura 5). Observa-se que a temperaturas mais baixas e a um pH básico houve um melhor rendimento na obtenção da AA. 
A polaridade do solvente utilizado, o tempo e a temperatura de extração são fatores que também podem influenciar na extração dos compostos antioxidantes, segundo Andreo et al. (2006).

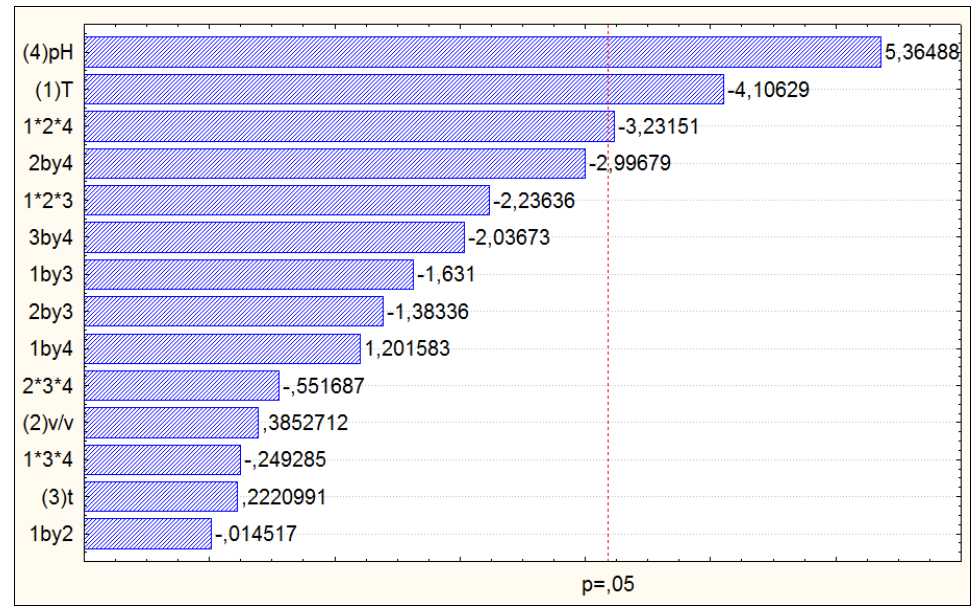

Figura 5 - Diagrama de Pareto para a atividade antioxidante

No Gráfico de Pareto para as antocianinas monoméricas totais (Figura 6) observou-se que os efeitos isolados de $\mathrm{pH}$ e tempo tiveram bastante influência na extração, assim como as interações da relação de solventes (2) com o tempo (3), a temperatura (1) com o pH (4) e a temperatura (1) com o tempo (3).

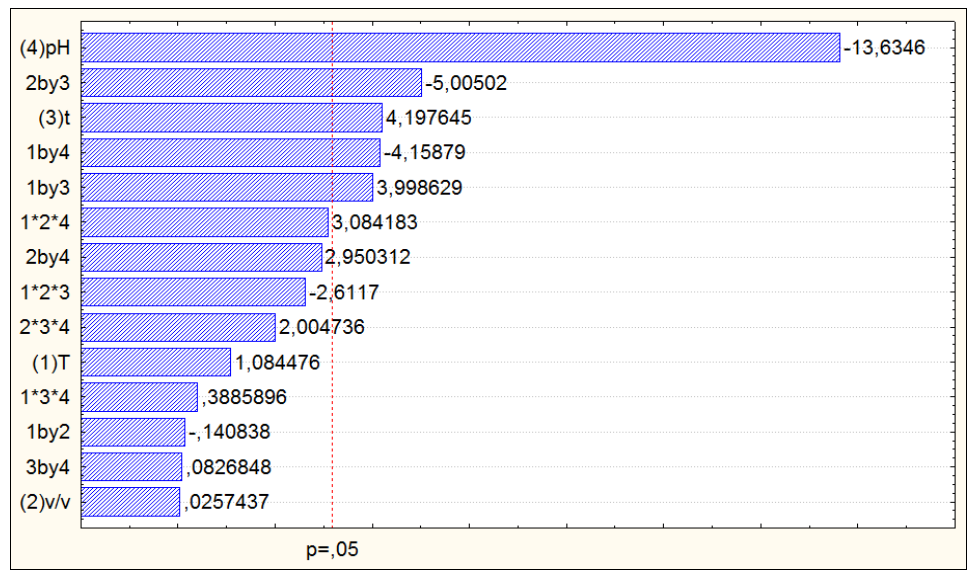

Figura 6 - Diagrama de Pareto para antocianinas monoméricas totais (AMT)

Observou-se que em tempos maiores e maiores concentrações de etanol há uma melhor obtenção de AMT (Figura 7a). Cacace et al. (2003), concluíram que a combinação de água e 
solventes orgânicos é geralmente a melhor opção para extração de compostos bioativos. Segundo os autores, o aumento da concentração de etanol reduz a constante dielétrica do solvente, diminuindo a solvatação das moléculas e, com isso, o aumento da sua difusão através da redução da interação com o solvente. Gruz et al. (2013) afirmam que a solubilidade desses compostos depende mais fortemente da polaridade por serem pigmentos hidrossolúveis. As figuras $7 b, c$ inferem que, as condições mais favoráveis para a extração da AMT, requerem o aumento do tempo e a redução do $\mathrm{pH}$ em temperaturas mais elevadas.

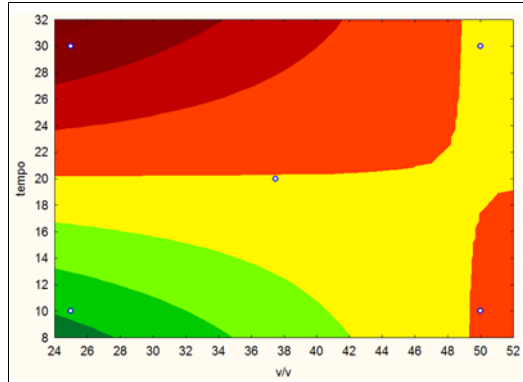

a)

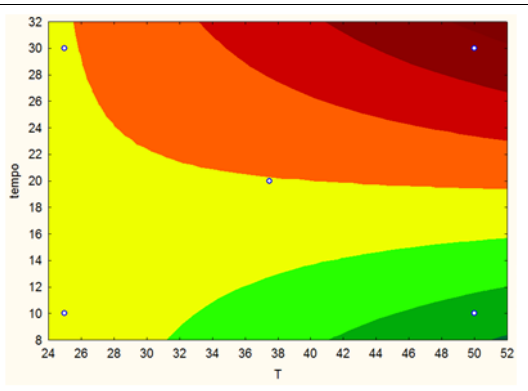

b)

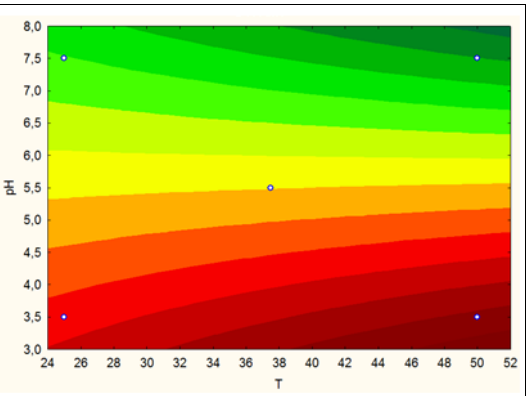

c)

Figura 7 - Curvas de níveis para AMT. Tempo versus a proporção do solvente (a), tempo versos a temperatura (b) e $\mathrm{pH}$ versus a temperatura (c)

\section{CONCLUSÃO}

Este trabalho analisou, através do planejamento experimental, os fatores que influenciam a extração do corante da castanhola. Com um planejamento fatorial $2^{4}$ (16 experimentos e 4 pontos centrais) foi possível se obter informações para melhorar o processo de obtenção da AA, CFT e AMT nos extratos.

De acordo com os valores dos coeficientes de determinação $\left(\mathrm{R}^{2}\right)$, cada modelo consegue explicar mais de $90 \%$ os valores observados. Conclui-se que o modelo linear é adequado.

Dentre todos os fatores estudados (temperatura, relação de solventes (água/etanol), tempo e $\mathrm{pH}$ ), o pH foi o que mostrou uma maior influência no processo de extração para obtenção de AA, CDT e AMT. Isto foi observado pelos Gráficos de Pareto para todas as variáveis respostas.

Assim, o presente estudo mostrou que, com controle das condições de operação no processo de extração do corante da castanhola, foi possível otimizar a obtenção dos compostos avaliados com a obtenção das condições ótimas de operação para AA $\left(25^{\circ} \mathrm{C}, 50 \%\right.$ de água, 10 min e $\mathrm{pH} 7,5)$, $\mathrm{CFT}\left(50^{\circ} \mathrm{C}, 50 \%\right.$ de água, 30min e $\left.\mathrm{pH} 3,5\right)$ e AMT $\left(25^{\circ} \mathrm{C}, 10 \%\right.$ de água, $30 \mathrm{~min}$ e $\mathrm{pH} 3,5$ ). 


\section{REFERÊNCIAS}

ANDREO, D.; JORGE, N. Antioxidantes naturais: técnicas de extração. Boletim do Centro de Pesquisa de Processamento de Alimentos, v.24, n.2, p.319-326, 2006.

AZEVEDO, J. C. S. Estratégias de obtenção do corante do jambo vermelho (Syzygium malaccense) e avaliação de sua funcionalidade. 2010. 101f. Dissertação (Mestrado em Engenharia Química) - Programa de Pós-Graduação em engenharia química, universidade Federal do Rio Grande do Norte, Natal. RN.

CACACE, J. E.; MAZZA, G. Mass transfer process during extraction of phenolic compounds from milled berries. J. of Food Eng., v.59, p.379- 389, 2003.

CHEPLICK, S.; KWON, YI.; BHOWMIK, P.; SHETTY, K. (2010). Phenolic-linked variation in strawberry cultivars for potential dietary management of hyperglycemia and related complications of hypertension. Bioresour Technol., 101: 404-413.

DUARTE-ALMEIDA, J. M. Avaliação da atividade antioxidante utilizando sistema $\beta$ caroteno/ácido linoléico e método de seqüestro de radicais DPPH. Ciên. e Tecn. de Alim., v. 26, n. 2, p. 446-452, abr./jun. 2006.

GRUZ A.P. G.; SOUZA C. G. S.; TORRES A. G.; FREITAS S. P.; CABRAL L. M. C. Recuperação de compostos bioativos a partir do bagaço de uva. Rev. Bras. Frutic. v. 35, n. 4, p. 1147-1157, Dezembro 2013.

GIUSTI, M.; WROLSTAD, R. E. Characterization and Measurement of Anthocyanins by UVVisible Spectroscopy. Curr. Protoc. in Food Anal. Chemistry, New York: John Wiley \& Sons 2001.

JING, P.; GIUSTI, M.M. Effects of Extraction Conditions on Improving the Yield and Quality of an Anthocyanin-Rich Purple Corn (Zea mays L.) Color Extract. J. Food Sci., 2007

TEIXEIRA, H. T. Composição química e perfil de ácidos graxos da castanha do fruto da castanhola (Terminalia Catappa Linn). 2010. 60 f. Dissertação (Mestrado em Engenharia de Alimentos) - Programa de Pós-Graduação em engenharia de alimentos, universidade estadual do sudoeste da Bahia, Campus de Itapetinga-BA.

SARTORI, J. A. S.; SCARLISE C.; BAPTISTA A. S.; LIMA R. B.; AGUIAR C. L. Parâmetros de influência na extração de compostos fenólicos de partes áreas da cana-de-açúcar com atividade antioxidante total. v. 29, n. 2, p. 297-307. Biosci. J. 2013. 